\title{
The Effects of Myofascial Trigger Point Injections on Nocturnal Calf Cramps
}

\author{
Do-Hyeong Kim, MD, Duck Mi Yoon MD, PhD, and Kyung Bong Yoon MD, PhD
}

Background: The purpose of this study was to elucidate the effects of injection at trigger points on pain and sleep disturbance in patients with nocturnal calf cramps (NCCs).

Methods: Patients with NCCs that occurred at least once per week and who had myofascial trigger points (MTrPs) on the gastrocnemius muscles were enrolled in the study for 9 months. At the first visit (T0), we measured the intensity of NCC pain on an 11-point numeric rating scale, recorded the frequency of NCCs, and calculated the Insomnia Severity Index (ISI). We then checked for MTrPs on the gastrocnemius muscles and injected 1-2 $\mathrm{mL}$ of $0.25 \%$ lidocaine into each of the trigger points. At 1 (T1), 2 (T2), and 4 (T3) weeks after the first visit, we repeated the process performed at T0.

Results: Twelve patients completed the treatment schedule and attended the follow-up visits. Mean values of the numeric rating scale pain score, frequency of cramps, and ISI declined significantly at T1, T2, and T3 compared with baseline (all $P<.01$ ). Of 12 patients, 10 had clinical insomnia before treatment, and this number decreased significantly to 3 patients at T2 and 1 patient at T3 $(P=.012$ and $P=.001$, respectively).

Conclusions: These preliminary data show that injection at MTrPs in patients with NCCs not only alleviated pain and reduced the frequency of cramps but also lessened the severity of insomnia as measured by the ISI. A larger randomized controlled trial is needed to confirm these findings and determine whether the effect lasts over the long term. (J Am Board Fam Med 2015;28:21-27.)

Keywords: Insomnia, Muscle Cramp, Myofascial Pain Syndromes

Nocturnal leg cramps are sudden, involuntary, painful contractions of the lower extremity in the nighttime, most often in the calf muscles, that gradually lessens. During a calf cramp, the affected muscles become firm and the foot and toes are held in plantarflexion. ${ }^{1,2}$ While cramps last from a few seconds to several minutes, pain and tenderness may remain for hours after the cramp itself ends. ${ }^{3}$ One third of adults experience nighttime calf muscle cramping, the prevalence increases as one ages, and $38 \%$ to $50 \%$ of elderly people suffer from

This article was externally peer reviewed.

Submitted 14 May 2014; revised 29 July 2014; accepted 5 August 2014.

From the Department of Anesthesiology and Pain Medicine, Anesthesia and Pain Research Institute, Yonsei University College of Medicine, Seoul, Korea.

Funding: none.

Conflict of interest: none declared.

Corresponding author: Kyung Bong Yoon, MD, PhD, Department of Anesthesiology and Pain Medicine, Anesthesia and Pain Research Institute, Yonsei University College of Medicine, 50-1, Yonsei-ro, Seodaemun-gu, Seoul 120-752, Korea (E-mail: kbyoon@yuhs.ac). night cramps. ${ }^{4,5}$ Athletes and pregnant women are also prone to cramps. ${ }^{6,7}$ Nocturnal leg cramps reportedly occur frequently in the calf muscles. ${ }^{8}$ In most subjects (73\%) the cramps occur only at night; $20 \%$ of patients report cramps during the day and night, and $7 \%$ have only daytime cramps. ${ }^{9}$ The etiology of nocturnal leg cramps is unclear, but diseases, including neuromuscular disease, diabetes mellitus, hypocalcemia, and peripheral vascular disease, and medications, such as oral contraceptives, diuretics, and steroids, are reported to predispose people to nocturnal leg cramps. ${ }^{1,3}$

Cramps are generally a benign and transient problem. Therefore patients do not usually feel anxious when nocturnal leg cramps occur with low frequency and as a sporadic condition. Frequent nocturnal leg cramps can, however, cause pain and significant distress. ${ }^{1,4}$ Although nonpharmacologic treatments, including stretching and massage, ${ }^{10}$ and pharmacologic treatments, including quinine sulfate, ${ }^{11}$ magnesium, ${ }^{12}$ and gabapentin, ${ }^{13}$ have been suggested for the management of nocturnal leg cramps, no treatment has yet demonstrated adequate efficacy and safety.,8 
Nocturnal leg cramps are a common problem that may adversely affect sleep. ${ }^{1}$ One study reported that patients with nocturnal calf cramps (NCCs) experienced sleep disturbance as well as pain and that NCCs disrupted the quality of sleep. ${ }^{2}$ However, studies considering sleep disturbance from the aspect of treatment efficacy are difficult to find. Prateepavanich et $\mathrm{al}^{14}$ revealed that trigger point $(\mathrm{TrP})$ injection into the gastrocnemius muscles was effective for NCCs in the aspects of pain intensity, frequency, and duration of cramps, but they did not look into the efficacy with regard to sleep disturbance. The purpose of this study was to elucidate the effects of $\operatorname{TrP}$ injection into the gastrocnemius muscles on pain and sleep disturbance in patients with NCCs.

\section{Methods}

After acquiring approval from the institutional review board of Severance Hospital, Yonsei University Health System (ref. 4-2012-0197), we registered this study at www.clinicaltrials.gov (identifier NCT01804556). Participants were enrolled in this study from our outpatient department for pain management between April and December 2013. Before enrollment, written informed consent was obtained from all participants. We studied male and female patients, aged 20 to 80 years, who visited our outpatient department for pain management of NCCs at least once per week. In this study a muscle cramp was required to occur exclusively at night while at rest and not be preceded by physical exercise. Patients with myofascial TrPs (MTrPs) on the gastrocnemius muscles were included in this study. According to criteria determined by Simons and Travell, ${ }^{15}$ a diagnosis of MTrPs of the gastrocnemius muscle requires the following: (1) a welldefined, tender, hypersensitive, palpable nodule located within a taut band of the upper gastrocnemius muscle; (2) a typical referred pain pattern; and (3) a local twitch response elicited by snapping palpation of the MTrP. To identify the nodule located within a taut band, the patient lies in a prone position and the clinician uses flat palpation-examination using finger pressure, proceeding across the muscle fibers at a right angle to their length while compressing them against a firm underlying structure, such as bone. A local twitch response is a transient contraction of essentially those muscle fibers in the tense band that are associated with MTrPs. The response is elicited by a sudden change of pressure in the MTrP, usually produced by transverse snapping palpation of the MTrP across the direction of the muscle fibers (taut band), like plucking violin strings. For all patients, a skilled clinician with 4 years' experience in diagnosing MTrPs performed the physical examinations (Figure $1 \mathrm{~A}$ and $\mathrm{B}$ ). $\mathrm{Pa}$ tients were excluded if they suffered from electrolyte disorders or congenital musculoskeletal disease or had taken sleeping pills or a sedative in the preceding 4 weeks. Patients also were excluded if they refused to be recruited, had other pain or disease requiring treatment on a preferential basis, were unable to communicate, or had cognitive impairment. Before the examination, we recorded patients' history of spine surgery and whether they were taking medications that can affect the occurrence of cramps, such as diuretics, statins, calcium channel blockers, and anticonvulsants. The patients were requested not to change their medication during the study period.

At the first visit (T0), we measured the pain intensity of NCCs using an 11-point numeric rating scale (NRS; $0=$ no pain, $10=$ the worst pain), recorded the frequency of NCCs (FC), and surveyed patients using the Insomnia Severity Index (ISI). The ISI is a brief self-report questionnaire assessing the nature, severity, and impact of insomnia. ${ }^{16} \mathrm{~A}$ higher score on the ISI indicates greater insomnia severity, ${ }^{17}$ and clinical insomnia is determined by a cutoff score of 15 on the ISI. ${ }^{18,19}$ This study recorded whether subjects had clinical insomnia, along with the ISI score. We then checked for MTrPs on the gastrocnemius muscles and injected 1 to $2 \mathrm{~mL}$ of $0.25 \%$ lidocaine into each of the $\mathrm{TrPs}^{14,20}$ (Figure 1C). One week later (T1), if the patients came to the hospital, we again recorded the NRS pain score, FC, and the ISI score; rechecked MTrPs; and repeated $\operatorname{TrP}$ injection only in cases in which MTrPs existed. If the patients did not come in, we surveyed the NRS pain score, FC, and the ISI over the phone. Two weeks after the first visit (T2) we repeated the process performed at T0. Four weeks after the first visit (T3) we surveyed the NRS pain score, FC, and the ISI face to face or over the phone.

\section{Statistical Analysis}

Sample size calculation was based on NRS pain score as the primary outcome. Previous study has demonstrated that the standard deviation (SD) of 
Figure 1. A: Locations of myofascial trigger points (TrPs) in the gastrocnemius muscle, where we identified myofascial TrPs in patients with nocturnal calf cramps in this study. B: Flat palpation, which compresses the muscle against the underlying bone to identify taut bands that harbor myofascial TrPs. C: Injection technique. The TrP is positioned between 2 fingers to prevent the TrP from sliding during injection. The fingers are pressed downward and apart to maintain pressure and ensure hemostasis. A 25-gauge, 1.5-inch needle is inserted between the fingers that have located the TrP. The needle penetrates the skin 1 to $2 \mathrm{~cm}$ away from the TrP so that the needle can approach it at an acute angle $\left(30^{\circ}\right)$ to the skin. The syringe, filled with $0.25 \%$ lidocaine, may be held between fingers of the injecting hand and thumb pressure used against the plunger. Ensure that the needle tip is positioned on the TrP, and then inject 1-2 mL of lidocaine.
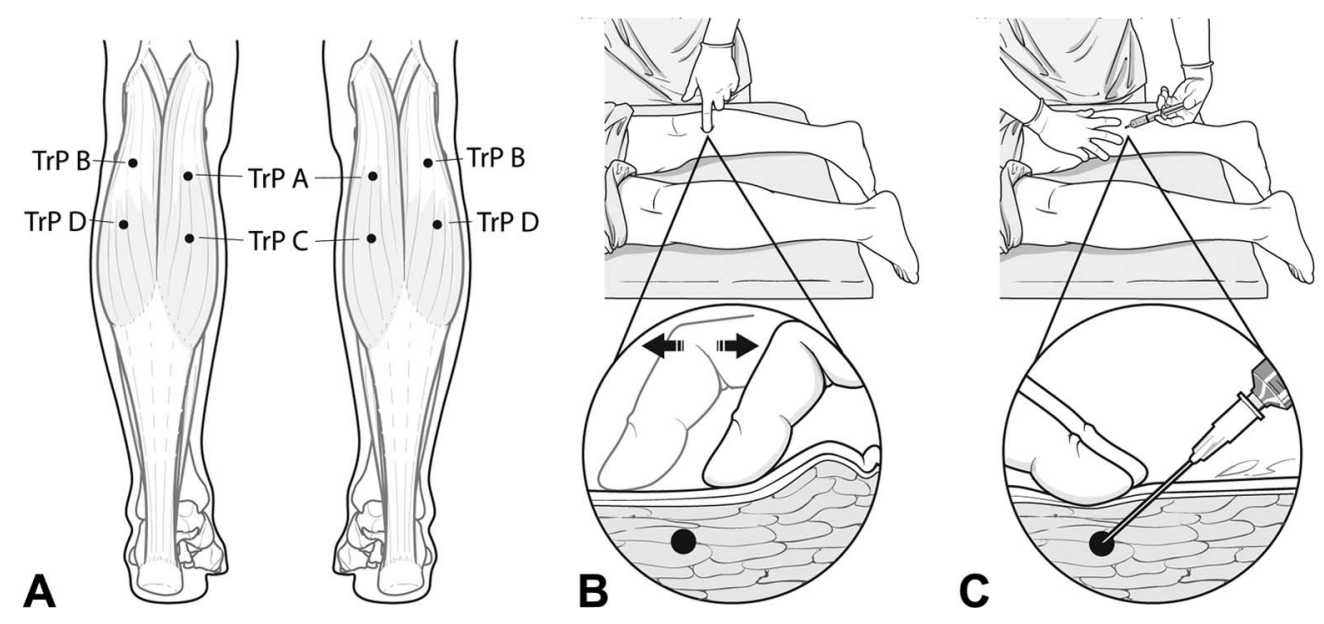

the NRS pain score is 2.4. ${ }^{14}$ Assuming a 5\% 2-tailed significance level $(\alpha=0.05)$ and power of $80 \%(\beta=0.20)$, a sample size of 11 patients was required to detect a mean difference of 2 in NRS pain score. ${ }^{21}$ To allow for possible dropouts, 13 patients were recruited at the beginning of the study. Continuous data are presented as mean \pm SD or median (range). The normality of the data distribution was assessed using the KolmogorovSmirnov test. Categorical data are reported as both frequency and percentage. For normally distributed data, comparisons of the variables with the baseline values at each time point were analyzed using repeated measures analysis of variance; for non-normally distributed data, the Friedman test and Wilcoxon signed rank test were used for analysis. Prevalence changes of clinical insomnia were assessed by the Fisher exact test. The statistical analysis was conducted using SPSS software version 18.0 for Windows (SPSS Inc., Chicago, IL). A $P$ value $<.05$ was considered statistically significant.

\section{Results}

A total of 13 patients met the inclusion criteria, 12 of whom completed the treatment schedule and attended a follow-up. Patient characteristics, calf cramps profile at baseline, and clinical presentations during the study period are described in Table 1. The average age of the patients was $63.1 \pm$ 10.2 years, and the average symptom duration was $36.0 \pm 13.5$ months. During the research period, none of the patients visited a hospital at T1, and only the patient interviews were assigned at $\mathrm{T} 3$ according to our study protocol. Therefore no MTrP injection was performed at T1 or T3. Five subjects had MTrP injection only once at T0 because they reported sufficient improvement of symptoms and they did not want additional treatment at T2. Five patients $(2,4,8,9$, and 11) reported that they were afraid to tense up their legs for fear of calf cramps.

Mean values of NRS pain score, FC, and the ISI score declined significantly at T1, T2, and T3 compared with baseline (all $P<.01$; Table 2). However, no significant differences were found between $\mathrm{T} 1$ and $\mathrm{T} 2, \mathrm{~T} 1$ and $\mathrm{T} 3$, and $\mathrm{T} 2$ and $\mathrm{T} 3$. Using an ISI score of 15 as a cutoff value for clinical insomnia, 10 of 12 patients had insomnia before treatment, and this number decreased significantly to 3 patients at T2 and 1 patient at T3 $(P=.012$ and $P=.001$, respectively; Figure 2$)$. There was a significant correlation between the difference in 


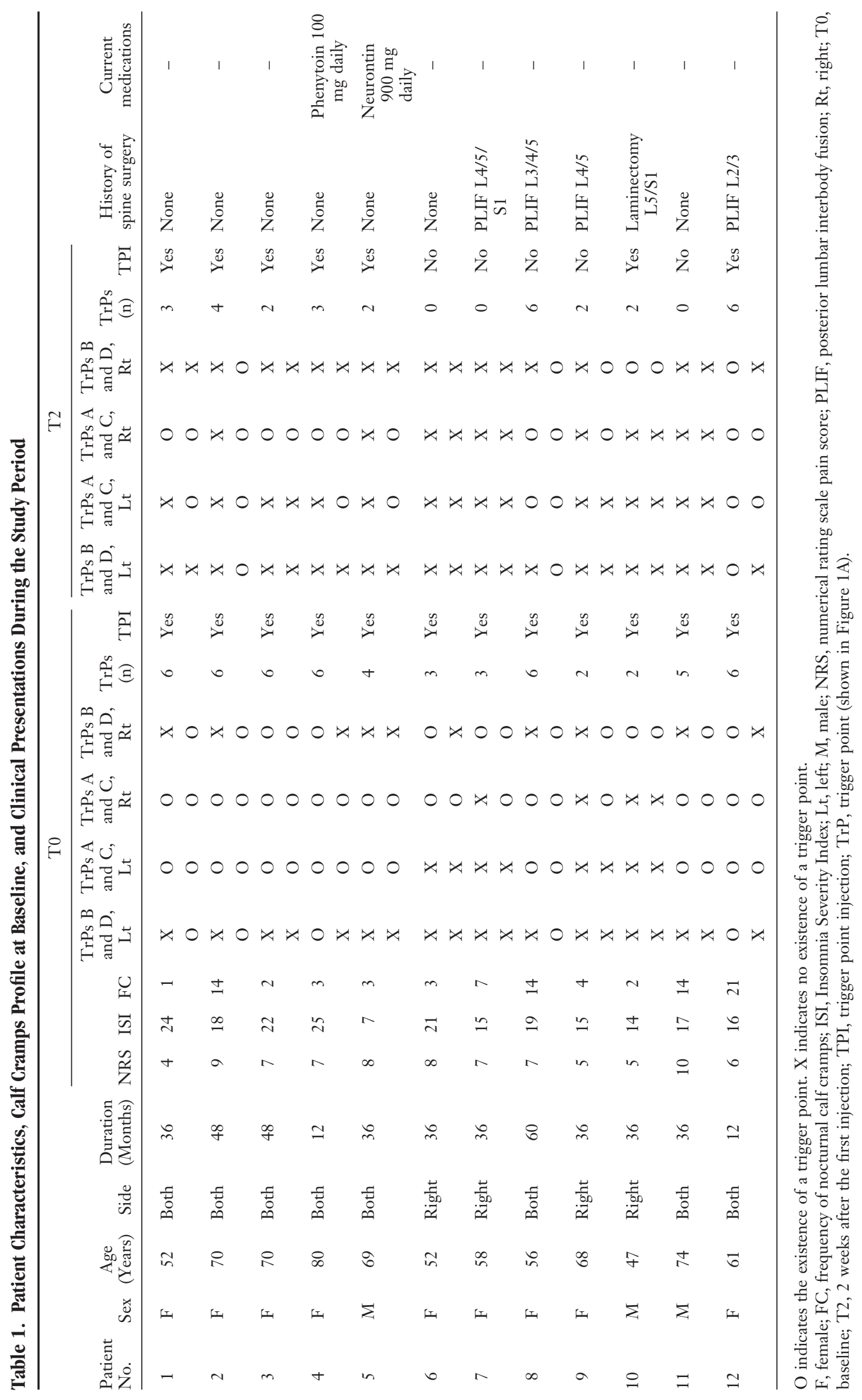


Table 2. Changes in Numerical Rating Scale Pain Score, Insomnia Severity Index, and Frequency of Nocturnal Calf Cramps During Treatment With Myofascial Trigger Point Injection(s)

\begin{tabular}{lcccc}
\hline & T0 & T1 & T2 & T3 \\
\hline NRS & $6.9 \pm 1.7$ & $4.8 \pm 2.0^{*}$ & $2.4 \pm 2.6^{*}$ & $2.2 \pm 2.2^{*}$ \\
FC & $3.5(1-20)$ & $1.0(0-7)^{\dagger}$ & $0.0(0-7)^{\dagger}$ & $0.5(0-2)^{\dagger}$ \\
ISI & $17.8 \pm 5.0$ & $12.8 \pm 4.6^{*}$ & $10.8 \pm 4.4^{*}$ & $8.9 \pm 3.7^{*}$ \\
\hline
\end{tabular}

Data are presented as mean \pm standard deviation or median (range).

${ }^{*} P<.01$ vs baseline (T0) using repeated measures analysis of variance.

${ }^{\dagger} P<.01$ vs T0 using Wilcoxon signed rank test.

FC, frequency of nocturnal calf cramps; ISI, Insomnia Severity Index; NRS, numerical rating scale pain score; T1, 1 week after the first injection; T2, 2 weeks after the first injection; T3, 4 weeks after the first injection.

number of MTrPs at T0 to T2 and the difference in NRS pain score at T0 to T3 (Spearman $\rho=$ 0.795; $P=.002$ ). During the study period, no subjects experienced complications or serious adverse events associated with the procedure.

\section{Discussion}

In this study we found that MTrP injection into the gastrocnemius muscle can reduce the severity of insomnia, as well as the FC and degree of pain among patients with NCCs. This finding suggests that sleep disturbance should also be considered in the treatment of NCCs.

A previous study showed that local anesthetic injection into the MTrPs of the gastrocnemius muscle in patients with NCCs is as effective as oral quinine, which used to be generally used for the prevention and treatment of nocturnal leg cramps. ${ }^{14}$ Whether MTrPs can be the source of NCCs, however, is not yet clear. According to evidence of the suggested pathophysiology of muscle cramps, cramps arise from spontaneous discharges of the motor nerves rather than from within the muscle itself. ${ }^{22}$ Other causes may include abnormal excitability of the terminal branches of motor axons ${ }^{23}$ and hyperactivity of the motor unit caused by disinhibition at the level of the spine. ${ }^{22}$ Roeleveld et al, ${ }^{24}$ however, showed that the temporal and spatial surface electromyographic characteristics of muscle cramps indicate that the cramp is initiated close to or even at the muscle fiber level. Ge et $\mathrm{al}^{25}$ concluded that increased nociceptive sensitivity at MTrPs may underlie the association between MTrPs and muscle cramps, and the motor endplate region and latent MTrPs thereabouts are potential candidates for the origin of muscle cramps based on the results of an experiment in which nociceptive input at latent MTrPs induced muscle cramps. Ge et al reported that treatment of MTrPs could provide therapeutic relief for muscle cramps. In the present study MTrP injection into the gastrocnemius muscle was effective in relieving the pain of NCCs, corresponding with the results of a study by Prateepavanich et al. ${ }^{14}$

Insomnia is a clinical condition characterized by difficulty initiating or maintaining sleep, accompanied by symptoms such as irritability or fatigue during wakefulness. ${ }^{26}$ In general, painful conditions and sleep disturbance may influence each other, ${ }^{27,28}$ and nocturnal episodes of pain in particular are reported to change sleep architecture, induce phases of arousal, and even trigger awakening. ${ }^{29}$ Hawke et $\mathrm{al}^{2}$ showed that NCCs had a substantial connection with reduced quality of sleep and, by extension, affected health-related quality of life. Blyton et $\mathrm{al}^{8}$ showed that most patients with NCCs were awakened by cramps and remembered cramp outbreaks at irregular times or in the middle

Figure 2. Prevalence of clinical insomnia determined by a cutoff score of 15 on the Insomnia Severity Index (ISI) at each time point during treatment with myofascial trigger point injection(s) for patients with nocturnal calf cramps. T0, baseline; T1, 1 week after the first injection; T2, 2 weeks after the first injection; T3, 4 weeks after the first injection. $* P<.05$ vs T0.

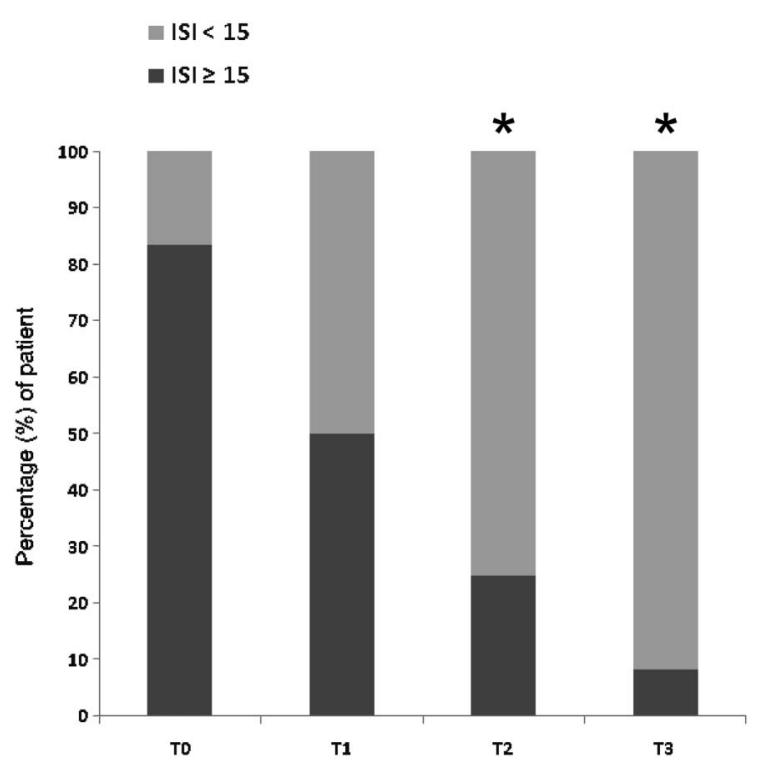


of sleep. In addition, NCCs seem to have a negative influence not only on sleep maintenance but also on sleep initiation. According to the aforementioned survey by Blyton et al, the most commonly suggested cause of cramps was sleeping position and movement from this position. Participants who identified the particular movements that induced cramp described plantarflexion of the ankle joint as the trigger. ${ }^{8}$ In the present study several patients $(2,4,8,9$, and 11) complained that they adopted an uncomfortable posture to avoid this movement for fear of calf cramps, and this physical strain may interrupt the initiation of sleep. Recent studies suggest that sleep fragmentation may be more critical to chronic pain than the quantity of sleep. ${ }^{29,30}$ Considering that night cramps occur at one or multiple points during the night, they may be a predisposing factor for sleep fragmentation, and this may cause a vicious cycle of night cramps and sleep disturbance. MTrP injection in patients with NCCs may therefore play a role not only in treating myofascial pain syndrome but also in stopping this vicious cycle.

The ISI has greater benefits than other available measures of clinical insomnia. Its specificity and its relative simplicity to administer and to score make it a popular insomnia screening tool in busy clinical settings. ${ }^{17,19}$ Although it is not used to definitively diagnose insomnia, the ISI was designed and validated with reference to the Diagnostic and Statistical Manual of Mental Disorders, Fourth Edition, criteria for insomnia, ${ }^{16}$ and the recommended cutoff indicates whether a sleep disorder justifies clinical attention. ${ }^{18}$ In addition, the ISI is sensitive to improvement in sleep over the course of both pharmacological and nonpharmacological treatments for insomnia. ${ }^{31}$ In this study, after MTrP injection, the mean values of the ISI decreased and the number of patients whose ISI score was $>15$ declined. Therefore we can conclude that MTrP injection into the gastrocnemius muscle for the treatment of NCCs can also be helpful in improving sleep disturbance. In addition, it indicates that in the case of treatment for pain accompanied by sleep disturbance, an accurate measurement of sleep disturbance is needed, not just a check of whether the patient is experiencing sleep disturbance.

This study has some limitations. First, a limited number of patients were available to be recruited for this study. The effects of MTrP injection in patients with NCCs were so distinct, however, that pain and sleep disturbance before and after treatment were significantly different despite the small number of subjects. Second, because of a lack of a control group in this study, the outcomes could not be considered definite findings. Further studies comparing the effect of MTrP injection and physical therapy with a drug such as gabapentin are needed. In addition, more study to verify the treatment effects of MTrP injection in patients with lumbar spinal canal stenosis, which is reported to include a high prevalence of nocturnal leg cramps, will be valuable. ${ }^{5}$ Third, we did not consider other factors that may have an effect on pain and sleep disturbance in patients with NCCs. Because the present research was a simple observational study on a small scale, it was difficult to handle many variables. Furthermore, even though no patients in this study had secondary causes of cramps, such as hypovitaminosis D, statin therapy, and cirrhosis, it is possible that local TrP injection produces a limited effect if the patients have such diseases or are taking medication. ${ }^{32}$ Therefore larger and controlled studies are needed.

\section{Conclusion}

MTrP injection into the gastrocnemius muscle in patients with NCCs not only alleviates pain and reduces the FC but also lessens the severity of insomnia as measured by the ISI. Considering the pilot nature of this study, however, its results could not be considered definite findings, and a larger study with a control group and a longer follow-up period is needed. As the clinical features of night cramps cause instantaneous severe pain and long-lasting soreness during sleep, rather than a persistent pain, measurement of sleep disturbance needs to be considered once a decision about the treatment effect of night cramps is made.

The authors thank Dong-Su Jang, MFA, Medical Illustrator, for his help with the illustrations in this article.

\section{References}

1. Monderer RS, Wu WP, Thorpy MJ. Nocturnal leg cramps. Curr Neurol Neurosci Rep 2010;10:53-9.

2. Hawke F, Chuter V, Burns J. Impact of nocturnal calf cramping on quality of sleep and health-related quality of life. Qual Life Res 2013;22:1281-6.

3. Riley JD, Antony SJ. Leg cramps: differential diagnosis and management. Am Fam Physician 1995;52:1794-8. 
4. Butler JV, Mulkerrin EC, O'Keeffe ST. Nocturnal leg cramps in older people. Postgrad Med J 2002;78: 596-8.

5. Matsumoto M, Watanabe K, Tsuji T, et al. Nocturnal leg cramps: a common complaint in patients with lumbar spinal canal stenosis. Spine (Phila Pa 1976) 2009;34(5):E189-94.

6. Vinciguerra G, Belcaro G, Cesarone MR, et al. Cramps and muscular pain: prevention with pycnogenol in normal subjects, venous patients, athletes, claudicants and in diabetic microangiopathy. Angiology 2006;57:331-9.

7. Dahle LO, Berg G, Hammar M, Hurtig M, Larsson L. The effect of oral magnesium substitution on pregnancy-induced leg cramps. Am J Obstet Gynecol 1995;173:175-80.

8. Blyton F, Chuter V, Burns J. Unknotting night-time muscle cramp: a survey of patient experience, helpseeking behaviour and perceived treatment effectiveness. J Foot Ankle Res 2012;5:7.

9. Naylor JR, Young JB. A general population survey of rest cramps. Age Ageing 1994;23:418-20.

10. Coppin RJ, Wicke DM, Little PS. Managing nocturnal leg cramps-calf-stretching exercises and cessation of quinine treatment: a factorial randomised controlled trial. Br J Gen Pract 2005;55:186-91.

11. Diener HC, Dethlefsen U, Dethlefsen Gruber S, Verbeek P. Effectiveness of quinine in treating muscle cramps: a double-blind, placebo-controlled, parallel-group, multicentre trial. Int J Clin Pract 2002; 56:243-6.

12. Roffe C, Sills S, Crome P, Jones P. Randomised, cross-over, placebo controlled trial of magnesium citrate in the treatment of chronic persistent leg cramps. Med Sci Monit 2002;8:CR326-30.

13. Serrao M, Rossi P, Cardinali P, Valente G, Parisi L, Pierelli F. Gabapentin treatment for muscle cramps: an open-label trial. Clin Neuropharmacol 2000;23: 45-9.

14. Prateepavanich P, Kupniratsaikul V, Charoensak T. The relationship between myofascial trigger points of gastrocnemius muscle and nocturnal calf cramps. J Med Assoc Thai 1999;82:451-9.

15. Simons DG, Travell JG, Simons LS. Travell \& Simons' myofascial pain and dysfunction: the trigger point manual. Vol 1, 2nd ed. Baltimore: Williams \& Wilkins; 1999.

16. Bastien CH, Vallières A, Morin CM. Validation of the Insomnia Severity Index as an outcome measure for insomnia research. Sleep Med 2001;2: 297-307.
17. Savard M, Savard J, Simard S, Ivers H. Empirical validation of the Insomnia Severity Index in cancer patients. Psychooncology 2005;14:429-41.

18. Smith S, Trinder J. Detecting insomnia: comparison of four self-report measures of sleep in a young adult population. J Sleep Res 2001;10:229-35.

19. Tang NK, Wright KJ, Salkovskis PM. Prevalence and correlates of clinical insomnia co-occurring with chronic back pain. J Sleep Res 2007;16:85-95.

20. Iwama H, Ohmori S, Kaneko T, Watanabe K. Water-diluted local anesthetic for trigger-point injection in chronic myofascial pain syndrome: evaluation of types of local anesthetic and concentrations in water. Reg Anesth Pain Med 2001;26:333-6.

21. Farrar JT, Young JP, LaMoreaux L, Werth JL, Poole RM. Clinical importance of changes in chronic pain intensity measured on an 11-point numerical pain rating scale. Pain 2001;94:149-58.

22. Miller TM, Layzer RB. Muscle cramps. Muscle Nerve 2005;32:431-42.

23. Layzer RB. The origin of muscle fasciculations and cramps. Muscle Nerve 1994;17:1243-9.

24. Roeleveld K, van Engelen BG, Stegeman DF. Possible mechanisms of muscle cramp from temporal and spatial surface EMG characteristics. J Appl Physiol 2000;88:1698-706.

25. Ge H, Zhang Y, Boudreau S, Yue S, Arendt Nielsen L. Induction of muscle cramps by nociceptive stimulation of latent myofascial trigger points. Exp Brain Res 2008;187:623-9.

26. Buysse DJ. Insomnia. JAMA 2013;309:706-16.

27. Lautenbacher S, Kundermann B, Krieg J. Sleep deprivation and pain perception. Sleep Med Rev 2006; 10:357-69.

28. Lautenbacher S. Pain, sleeping problems and their many relatives. Pain 2012;153:1138.

29. Lavigne G, Brousseau M, Kato T, et al. Experimental pain perception remains equally active over all sleep stages. Pain 2004;110:646-55.

30. Smith MT, Edwards R, McCann UD, Haythornthwaite JA. The effects of sleep deprivation on pain inhibition and spontaneous pain in women. Sleep 2007;30:494-505.

31. Morin CM, Bastien C, Guay B, Radouco Thomas M, Leblanc J, Vallières A. Randomized clinical trial of supervised tapering and cognitive behavior therapy to facilitate benzodiazepine discontinuation in older adults with chronic insomnia. Am J Psychiatry 2004;161:332-42.

32. Kuan T. Current studies on myofascial pain syndrome. Curr Pain Headache Rep 2009;13:365-9. 